\title{
Editorial: Mesenchymal Stem Cells and Interactions With Scaffolds - Biomaterials in Regenerative Medicine: From Research to Translational Applications
}

\author{
Francesco De Francesco* \\ Hand Surgery Unit, Department of Plastic Reconstructive Surgery, Azienda Ospedaliero Universitaria Ospedali Riuniti, \\ Ancona, Italy
}

Keywords: regenerative medicine, adipose stem cells, scaffold, translational applications, mesenchymal stem cells

Editorial on the Research Topic

Mesenchymal Stem Cells and Interactions With Scaffolds - Biomaterials in Regenerative Medicine: From Research to Translational Applications

\section{OPEN ACCESS}

Edited and reviewed by: Atsushi Asakura, University of Minnesota Twin Cities, United States

${ }^{*}$ Correspondence: Francesco De Francesco fran.defr@libero.it; francesco.defrancesco@ ospedaliriuniti.marche.it

Specialty section: This article was submitted to

Stem Cell Research,

a section of the journal Frontiers in Cell and Developmental Biology

Received: 21 May 2019 Accepted: 28 August 2019 Published: 12 September 2019

Citation:

De Francesco F (2019) Editorial: Mesenchymal Stem Cells and Interactions With Scaffolds Biomaterials in Regenerative Medicine: From Research to Translational Applications.

Front. Cell Dev. Biol. 7:193. doi: $10.3389 /$ fcell.2019.00193
It can be estimated that 1,500,000 patients in Europe undergo soft tissue reconstruction each year, and of these patients, about $20 \%$ undergo an experience of a loss of function despite reconstruction and, about 30,000 patients in Europe are suffering from donor site morbidity relating to flap reconstructions. In a defect, the different missing tissues involved have specific functions and their replacement is often quite difficult. For example, the closure of a defect is commonly associated with the transfer of tissue (e.g., a flap), which may not fully restore the unique function of the lost part. Also, each tissue transfer is associated with donor site morbidity, the most important being scars, infection and loss of function.

The ability to regenerate tissues is attributable to a pool of undifferentiated cells capable of replacing damaged cells in order to guarantee the integrity of the organism. The main source of stem cells from adult is the bone marrow (BMSC, Bone Marrow Stromal Cells) can easily be obtained from its stroma. A particularly interesting source of mesenchymal stem cells is represented by stem cells that can be isolated from adipose tissue (ASCs, Adipose Stem cells; Zuk et al., 2002; De Francesco et al., 2009). The ASCs have the potential to differentiate into bone (Tajma et al., 2018), cartilage (Szychlinska et al., 2017), skeletal muscle (Desiderio et al., 2013), fat (Ferraro et al., 2012), and other tissue (Planat-Benard et al., 2004; Tobita et al., 2008) when cultivated under specific conditions. The mesenchymal cells of adipose tissue are similar to bone marrow in the treatment of various tissue pathologies and therefore represent an important source for autologous cellular therapies. Fellows et al. explore the challenges associated with cartilage repair and regeneration using MSC-based cell therapies, focusing on cells capable of producing stratified hyaline-like articular cartilage regeneration. Moreover, Senesi et al. stated that ASCs play an important role in the treatment of osteoarthritis and the authors compare the mechanical and exymathic procedure to isolate the stromal vascular fraction from adipose tissue. In this paper, the authors showed that the mechanical procedures yielded no significant difference in cell viability and cluster differentiation expression to enzymatic procedure. Currently, the use of mechanical procedures for the isolation of the SVF from adipose tissue has become of vital importance. Especially for the requirements of minimal tissue manipulation and the impossibility of using collagenase. 
About that, Dessels et al. stated that ASCs must be used with good manufacturing procedures (GMPs) in humans and therefore fetal bovine serum, normally used in vitro research, can not be used. Then, for clinical trial research, ASCs must be expanded in vitro using xeno-free products and human blood-derived alternatives. In addiction, Purpura et al. collected adipose tissue using a water-jet assisted liposuction in order to preserve an high cell viability for the immediate use in the field of plastic and reconstructive surgery.

In addition to ASCs, the large family of mesenchymal stem cells also includes cells from oral and dental tissue. In this context, human periapical cysts mesenchymal stem cells (hPCy-MSCs) have been studied by Tatullo et al. This cells exhibit extensive proliferative potential, cell surface marker profile, and the ability to differentiate into various cell types.

Tissue engineering (TE), a collection of technologies combining biomaterials and stem cells, provides the tools for regenerative medicine (RM) and is expanding tremendously from biomaterial science toward a genuine multidisciplinary area, integrating biology, medicine, and various engineering sciences (Langer and Vacanti, 2016). Rodriguez y Baena et al. evaluated, in this paper, the ability of autologous periosteumderived micrografts in combination with a new biomaterials such as poly(lactic-co-glycolic acid) (PLGA) supplemented with hydroxyl apatite (HA) for bone augmentation in the sinus lift procedure. In this manner, the authors showed an increased percentage of vital mineralized tissue in the treated group respect to the control, confirmed by histological analysis and $\mathrm{Rx}$ evaluation. The use of biomaterial scaffolds selected in an appropriate way, together with growth factors can significantly improve survival and differentiation of transplanted stem cells. The biomaterial/scaffold itself, actually, may be an ideal delivery vehicle of chemical factors.

\section{REFERENCES}

De Francesco, F., Tirino, V., Desiderio, V., Ferraro, G., D’Andrea, F., Giuliano, M., et al. (2009). Human CD34/CD90 ASCs are capable of growing as sphere clusters, producing high levels of VEGF and forming capillaries. PLoS ONE 4:e6537 doi: 10.1371/journal.pone.0006537

Desiderio, V., De Francesco, F., Schiraldi, C., De Rosa, A., La Gatta, A., Paino, F., et al. (2013). Human $\mathrm{Ng}^{2+}$ adipose stem cells loaded in vivo on a new crosslinked hyaluronic acid-Lys scaffold fabricate a skeletal muscle tissue. J. Cell Physiol. 228, 1762-1773. doi: 10.1002/jcp.24336

Ferraro, G. A., De Francesco, F., Nicoletti, G., Paino, F., Desiderio, V., Tirino, V., et al. (2012). Human adipose $\mathrm{CD} 34^{+} \mathrm{CD} 90^{+}$stem cells and collagen scaffold constructs grafted in vivo fabricate loose connective and adipose tissue. J. Cell. Biochem. 1145, 1039-1049. doi: 10.1002/jcb.24443

Langer, R., and Vacanti, J. (2016). Advances in tissue engineering. J. Pediatr. Surg. 51, 8-12. doi: 10.1016/j.jpedsurg.2015.10.022

Planat-Benard, V., Silvestre, J. S., Cousin, B., André M., Nibbelink, M., Tamarat, R., et al. (2004). Plasticity of human adipose lineage cells toward endothelial cells: physiological and therapeutic perspectives. Circulation 109, 656-663. doi: 10.1161/01.CIR.0000114522.38 265.61

Szychlinska, M. A., Castrogiovanni, P., Nsir, H., Di Rosa, M., Guglielmino, C., Parenti, R., et al. (2017). Engineered cartilage regeneration from
We know from these studies and from many other published studies, that the MSCs have been used in a significant number of clinical trials with a good regenerative effect. Despite this, the functional role of these cells in tissue regeneration is not yet fully understood. In a review, Lunyak et al. demonstrated that MSCs have an autocrine and paracrine properties on the regulation of immune system and induce changes in the tissue microenvironment with numerous therapeutic effects on promoting repair and regeneration.

The current Research Topic will focus on the regeneration, repair, and rebuilding of tissues combining implantable biocompatible materials with stem cell technologies. Although numerous breakthroughs in stem cell research have been made thus far, their success and applicability in clinical trials remains to be ascertained. A number of issues will be important for the advancement of regenerative medicine as a field. First, stem cells, whether isolated from adult tissue or induced, will often require tight control over their behavior to increase their safety profile and efficacy after transplantation. Second, the creation of microenvironments, often modeled on various stem cell niches that provide specific cues, including morphogens and physical properties, or have the capacity to genetically manipulate target cells, will likely be key to promoting optimal regenerative responses from therapeutic cells. In the last decade several skills were acquired about the isolation, the morphological characteristics and the differentiation potential of adult stem cells in vitro.

\section{AUTHOR CONTRIBUTIONS}

FD contributed conception and design of the study, wrote the first draft of the manuscript, wrote sections of the manuscript, read, and approved the submitted version.

adipose tissue derived-mesenchymal stem cells: a morphomolecular study on osteoblast, chondrocyte and apoptosis evaluation. Exp. Cell Res. 357, 222-235 doi: 10.1016/j.yexcr.2017.05.018

Tajma, S., Tobita, M., and Mizuno, H. (2018). Current status of bone regeneration using adipose-derived stem cells. Histol. Histopathol. 33, 619-627. doi: 10.14670/HH-11-942

Tobita, M., Uysal, A. C., Ogawa, R., Hyakusoku, H., and Mizuno, H. (2008). Periodontal tissue regeneration with adipose-derived stem cells. Tissue Eng. Part A. 14, 945-953 doi: 10.1089/ten.tea.2007.0048

Zuk, P. A., Zhu, M., Ashjian, P., De Ugarte, D. A., Huang, J. I., Mizuno, H., et al. (2002). Human adipose tissue is a source of multipotent stem cells. Mol. Biol. Cell. 13, 4279-4295. doi: 10.1091/mbc.e02-02-0105

Conflict of Interest Statement: The author declares that the research was conducted in the absence of any commercial or financial relationships that could be construed as a potential conflict of interest.

Copyright (C) 2019 De Francesco. This is an open-access article distributed under the terms of the Creative Commons Attribution License (CC BY). The use, distribution or reproduction in other forums is permitted, provided the original author(s) and the copyright owner(s) are credited and that the original publication in this journal is cited, in accordance with accepted academic practice. No use, distribution or reproduction is permitted which does not comply with these terms. 